\title{
Alzheimer's Disease and Glaucoma: Imaging the Biomarkers of Neurodegenerative Disease
}

\author{
Denise A. Valenti \\ Vision Care, 62 Forest Avenue Quincy, MA 02169, USA \\ Correspondence should be addressed to Denise A. Valenti, deniseavalenti@gmail.com
}

Received 29 September 2010; Accepted 8 November 2010

Academic Editor: Adam S. Fleisher

Copyright () 2010 Denise A. Valenti. This is an open access article distributed under the Creative Commons Attribution License, which permits unrestricted use, distribution, and reproduction in any medium, provided the original work is properly cited.

Imaging through the visual system in Alzheimer's disease, with the technology currently in widespread use for the diagnosis and management of eye disease such as glaucoma and macular degeneration, is proving to be promising. In vivo cross-section imaging during an annual comprehensive eye exam has been available for a decade for glaucoma and macular degeneration, and this same imaging, using Optical Coherence Tomography, has been demonstrated to show deficits specific to AD and mild cognitive impairment. These deficits are in the form of nerve fiber layer tissue drop out in the retina and optic nerve. The retrograde loss of nerve fiber layer tissue in the retina and optic nerve may be an early biomarker of AD, and these deficits in the nerve fiber layer of the retina and optic nerve may be the earliest sign of $\mathrm{AD}$, even prior to damage to the hippocampal region that impacts memory.

\section{Introduction}

Biomarkers for the early diagnosis, management, and treatment of Alzheimer's disease (AD) are important. In vivo cross-section imaging during an annual comprehensive eye exam with a dilated pupil has been available for a decade for glaucoma and macular degeneration, and this same imaging, using Optical Coherence Tomography (OCT), has been demonstrated to show deficits specific to $\mathrm{AD}[1-7]$ and mild cognitive impairment (MCI) [2]. These deficits are in the form of nerve fiber layer tissue drop out in the retina and optic nerve. The retrograde loss of nerve fiber layer tissue in the retina and optic nerve may be an early biomarker of $\mathrm{AD}$, and these deficits in the nerve fiber layer of the retina and optic nerve may be the earliest sign of $\mathrm{AD}$, even prior to damage to the hippocampal region that impacts memory. The OCT technology used to identify nerve fiber layer defects in $\mathrm{AD}$ reported in the literature is an older version often referred to as Time Domain-OCT. Time DomainOCT (Time-OCT) only resolved the retina to approximately ten microns, whereas newer clinical OCT imaging systems High Definition/Resolution/Fourier/Spectral Domain-OCT (HDR-OCT) can resolve the retina to five microns or less, acquire images faster, reregister retinal location, and does not require the dilation of the pupils of the eye. Faster acquisition is important for patients with cognitive impairment such as $\mathrm{AD}$. The ability to reregister exact location enables more accurate followup and longitudinal study.

OCT is similar to ultrasound, but instead of measuring echoed sound waves, it measures the echoed time delays of a reflected light beam. As the beam is scanned, there is a cross-sectional image generated. The OCT used for ocular imaging has a fiber optic delivery system coupled with a slitbiomicroscope. The patient sits with their head positioned in a chin rest and the experience is similar to having a photograph taken of the eye. This type of imaging can easily be incorporated into annual eye exams for those with $\mathrm{AD}$.

\section{History}

The first published reports of imaging in vivo retinal tissue with OCT were in $1993[8,9]$, and the first reports of OCT use showing significant deficits in the eyes of participants with AD were in 2001 [5]. The technology has evolved considerably since these early works were published. OCT technology and its application in the eye had origins in the Department of Electrical Engineering and Computer Science at Massachusetts Institute of Technology where James G. Fujimoto, Ph.D., a professor in computer sciences 
and electrical engineering along with his students had collaborated with Carmen A. Puliafito, MD who at the time was affiliated with the Massachusetts Eye and Ear Infirmary (MEEI) [10]. Another student working with Dr. Fujimoto contributed with the idea to generalize axial scanned images to transverse images [10] and published the precursor images of ex vivo retina [11] and these are considered to be the first illustration of OCT use. Joel S. Schuman, MD was a glaucoma fellow at MEEI in 1989 and was doing work in the laboratory of Dr. Puliafito. He eventually worked closely with the New England Eye Center at Tufts Medical School where over 10,000 patients were imaged with the initial prototype [10]. Clinical studies took place between 1994 and 1995 and Carl Zeiss Meditec made available the first commercial instrument in 1996, the Stratus-OCT [10]. Optovue, Carl Zeiss Meditec, Heidelberg Engineering, Topcon Medical Systems, and Bioptigen are some of the other companies that have developed imaging technology using the HDR-OCT systems.

All publications to date, using OCT technology in the study of AD, have used the Stratus-OCT from Carl Zeiss Meditec which was the Time-OCT. The parameters are longitudinal axial resolution $<10$ microns in tissue, transverse resolution 20 microns in tissue, scan speed $400 \mathrm{~A}$ scans per second, longitudinal depth $2 \mathrm{~mm}$ in tissue, and an optical source of superluminescent diode at 840 nanometers [12]. The newer HDR-OCT from Carl Zeiss Meditec; the Cirrus/HD-OCT has improvements that will make the technology more effective in $\mathrm{AD}$. Among the improvements is the ability to reregister the images assuring more precise, repeat measurements for longitudinal studies. The parameters are longitudinal axial resolution $<5$ microns in tissue, transverse resolution 15 microns in tissue, scan speed 27,000 scans per second, longitudinal depth $2 \mathrm{~mm}$ in tissue, and an optical source of superluminescent diode at 840 nanometers [13]. Figure 1 shows Stratus-OCT images of the nerve fiber layer in a normal age control participant that was age matched to the AD participant, a participant with $\mathrm{AD}$ and a participant with both glaucoma and Parkinson's disease (not age matched).

While it is likely that HDR-OCT technology will become an integral part of the diagnosis and management of $\mathrm{AD}$, differentiating the optic nerve fiber losses and retinal changes from common age-related ocular disease, and in particular glaucoma will remain clinically demanding. The two diseases, AD and glaucoma, impact the same visual pathways early in their disease processes. Additionally both diseases can result in subtle changes in the functional aspects of vision.

The OCT imaging picks up the deficits related to $\mathrm{AD}$ as structural drop out due to retrograde degeneration from the brain tissue in visual processing areas such as the visual association area, Brodmann 19. Further, the degeneration in the visual association area may be one of the earliest brain tissues damaged by $\mathrm{AD}$, even before the memory/hippocampal areas. That neurodegenerative pathology of $\mathrm{AD}$ originates in the visual association area is indicated in the studies by McKee and colleagues of autopsied brains in the Framingham Heart Study [14].
McKee and colleagues found that in the visual associationBrodmann 19 area of over $50 \%$ of those that had been classified as being cognitively normal, having no memory impairments, there were findings related to $\mathrm{AD}$ such as beta amyloid and neurofibrillary tangles. Similar structural findings were commonly associated with Alzheimer's disease. In some of the cognitively normal cases, there was no ADrelated pathology in the hippocampus area which processes memory, but there was AD related pathology in the Visual Association-Brodman 19 [14].

Losses in visual function attributed to $\mathrm{AD}$ have many aspects in common with the functional losses in glaucoma. Both diseases show structural changes early in their course in the visual cortex $[15,16]$ and lateral geniculate nucleus [1719]. The ability to detect motion is reduced in $\operatorname{AD}[20,21]$; there are visual field defects [22, 23]; contrast sensitivity deficits in the lower spatial frequencies have been reported in participants with $\mathrm{AD}$ [24]. Glaucoma also impacts lower spatial frequencies in contrast sensitivity and produces visual field defects [25].

\section{Glaucoma and AD [26]}

There is a more aggressive destruction of structure and function when patients have both AD and glaucoma [27]. Glaucoma is a painless and insidious disease process and often goes undetected. Glaucoma impacts up to three million Americans, but only one half of those with glaucoma are aware they have it. Glaucoma is responsible for $9-12 \%$ of blindness in the United States. Glaucoma is the leading cause of blindness among African Americans and Hispanics. Those over the age of 60 of any race are at greater risk of developing glaucoma and it is estimated that $8 \%$ of those over the age of 80 have elevated IOP. Glaucoma and AD affect the magnocellular visual processing. The magnocellularmediated visual functions are those that are not easily assessed during conventional eye examinations such as motion processing and contrast sensitivity function. There are losses in the magnocellular layers of the lateral geniculate nucleus in glaucoma $[17,18]$. The lateral geniculate nucleus neurons early on in the glaucoma disease process show significant shrinkage and are observed even before nerve fiber loss in the optic nerve with conventional direct viewing methods [28, 29]. HDR-OCT likely identifies these early losses. A single case study reported in the literature by Gupta and colleagues [15] regarding changes in the visual cortex in human glaucoma indicated reductions in autopsied tissue in the area below the calcarine sulcus compared to normal control. This region of change corresponded to an area of superior visual field loss that had been identified in the participant prior to death. Structural defects specific to the magnocellular visual pathway have also been identified in individuals with $\mathrm{AD}$ even in brain areas devoid of plaques and neurofibrillary tangles. The magnocellular visual pathway shows signs of individual cell drop out in the primary visual cortex of AD individuals [16]. Neurofibrillary tangles and amyloid plaques have been identified in the lingual and cuneal gyri of participants with $\mathrm{AD}$, and these correlate with 
the incidence of functional visual field loss in $\mathrm{AD}$ participants [22]. The magnocellular layers in the lateral geniculate nucleus have been shown to have plaques associated with $\mathrm{AD}$ [19]. The visual association area processes signals from the magnocellular visual pathway.

Glaucoma and AD have disruptions in circadian function [30]. "Sundowning" may be caused by disruptions in the circadian rhythm of AD patients. Sundowning is a general term describing symptoms such as sleep disturbance, nocturnal delirium, disorientation at the onset of darkness, night-time activity, and agitation [31]. Disturbances in the circadian rhythm occur in $\mathrm{AD}$ patients, with rates ranging from $12 \%$ [32] to $25 \%$ [33]. $\mathrm{AD}$ is associated with changes in the suprachiasmatic nucleus $[34,35]$.

The eye's intraocular pressure follows a circadian rhythm with increases in intraocular pressure at night [36-39]. There are reports that found greater fluctuation and range in intraocular pressure in individuals with glaucoma compared to healthy control individuals $[39,40]$. Studies by Liu and colleagues [38] found that the larger the diurnal variation and fluctuation of intraocular pressure, the greater the risk for structural damage due to glaucoma. Intraocular fluctuation may not be a risk factor, but actually an early symptom of glaucoma, as the disease impacts the optic nerve and the axons leading to brain structures processing circadian function. Light has a strong influence on the circadian system and the timing and duration of exposure to daylight can impact circadian phases, melatonin suppression, physiological responses, and alertness [41]. A previously unknown photoreceptor that was dissimilar to both rod cells and cone cells was identified by Berson and colleagues [42]. These cells are retinal ganglion cells that directly innervate the suprachiasmatic nucleus and are reactive to light even when all synaptic input from rod cells and cone cells is blocked. Damage anywhere along the visual pathways that process functions related to the circadian retinal cells has potential to disrupt circadian rhythms, and these structural deficits will result in further reduction of the nerve fiber layers in the retina as measured by OCT. This would be the case whether the site of origin is in the brain such as in $\mathrm{AD}$ or in the optic nerve as in glaucoma.

Studies have identified a higher incidence of glaucoma among those residing in nursing homes. One such study found a higher rate of glaucoma among 112 residents with $\mathrm{AD}$ compared to 774 residents without AD. The diagnosis of glaucoma was based on visual field defects or optic nerve cupping observed during eye examinations. While there may be field defects in $\mathrm{AD}$, there is generally no optic nerve cupping. Optic nerve cupping is a biomarker related to glaucoma. The rate of glaucoma was $25.9 \%$ for the $\mathrm{AD}$ patients and $5.2 \%$ for the control nursing home group [43]. The optic nerve may be less resistant to elevated intraocular pressure levels with $\mathrm{AD}$. A record review in a large glaucoma clinic identified seven patients with elevated intraocular pressures or ocular hypertension that initially had normal visual fields and normal optic discs and had also been diagnosed within one year of also having AD. Among these patients, there was a more glaucomatous optic neuropathy which was much more rapid compared to other glaucoma patients [27]. This has also been reported to be the case for Parkinson's disease also [44]. Figure 1 shows the nerve fiber layer deficits identified with Time-OCT in a participant that had both glaucoma and Parkinson's disease. Figure 1 also shows the same participants optic nerve, demonstrating both nerve fiber loss and optic nerve cupping.

A study found a higher rate of glaucoma among $\mathrm{AD}$ patients compared to non-AD patients. In their study of 172 patients with $\mathrm{AD}$ in Japan compared to 176 age matched controls, Tamura and colleagues [45] found those with $\mathrm{AD}$ had a rate of glaucoma of $23.8 \%$ compared to only $9.9 \%$ of the age-matched controls having glaucoma. If there are two neurodegenerative processes such as glaucoma and $\mathrm{AD}$ affecting one of the major relay centers for visual function, it is easy to appreciate that the loss in function can be substantial.

As $\mathrm{AD}$ progresses causing substantial reductions in brain volumes, there may be additional impact on the visual system creating abnormal pressure gradients at the back of the eye along the nerve. This can cause stress and damage to the lamina cribrosa, the supportive tissue to the optic nerve. When there is a lower pressure on the posterior nerve such as was found in a study of cerebrospinal fluid pressure by Berdahl and Allingham, there is a significantly higher rate of glaucoma [46]. In their study, the average cerebrospinal fluid pressure was 33\% lower in those with glaucoma compared to those without a diagnosis of glaucoma. Morgan and colleagues theorize that when the cerebrospinal fluid pressure is low, there is an influence on subarachnoid space. Because of the space location posterior to the globe and nerve and its pressure relationships to intracranial, retrolaminar and translaminar tissue it is as if there is damage much like would occur with an elevation of pressure inside the eye [47]. There is increasing evidence that low cerebrospinal fluid pressure causes glaucoma like damage [48-50]. Wostyn and colleagues have had long standing theories regarding changes in cranial pressure and the relationship of the stress of change to neurodegenerative diseases including $\mathrm{AD}$ and glaucoma [51], and the recent findings related to reduced cerebrospinal fluid and glaucoma are supportive of this. Wostyn reports in a study that a subgroup of $\mathrm{AD}$ participants had low cerebrospinal fluid pressures and he attributed this to brain atrophy. He further theorizes, as did Morgan and colleagues, that this produces an abnormal high translamina pressure difference and this is what leads to the higher incidence of glaucoma among those with $\mathrm{AD}$ [52].

The relationship between $\mathrm{AD}$ and glaucoma remains poorly understood. Published studies look at more advanced dementia in already diagnosed $\mathrm{AD}$ and residents in nursing homes with $\mathrm{AD}$. To date there are no published studies investigating glaucoma in Mild Cognitive Impairment or in mild AD. This still leaves the possibility that the two diseases are unrelated, but because they affect the same axonal structures and visual pathways the result is an expression of the vision losses related to glaucoma at an earlier stage of the disease. It is estimated that thirty to fifty percent of ganglion cells and fibers in the visual pathway are damaged 

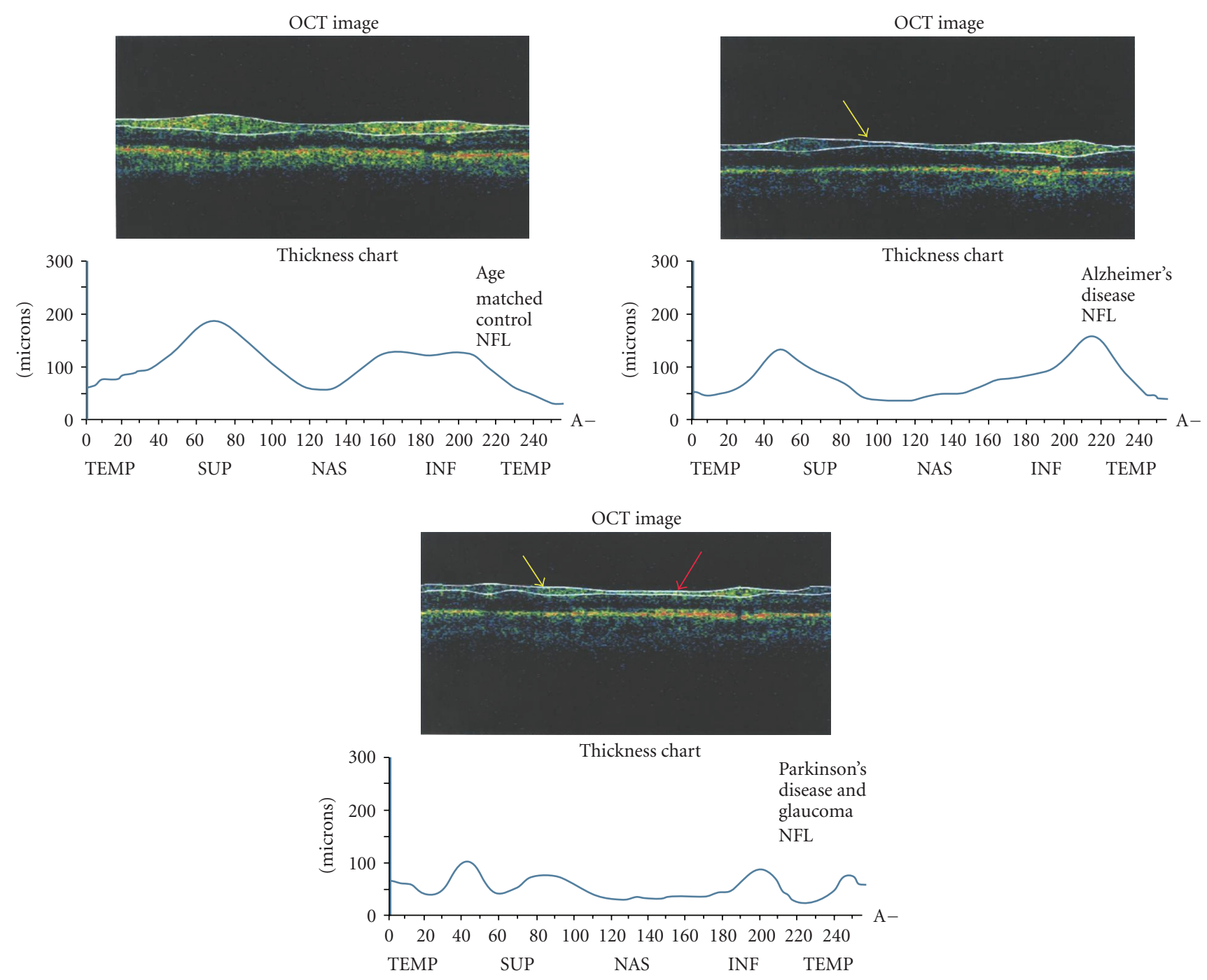

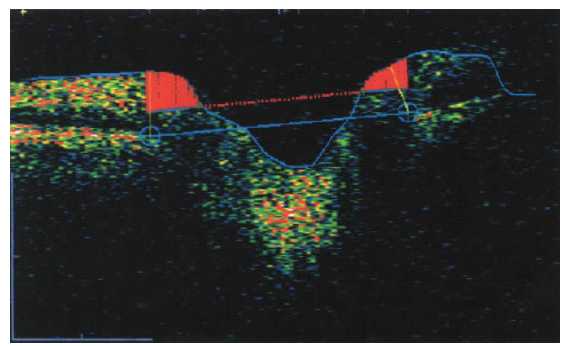

Normal nerve

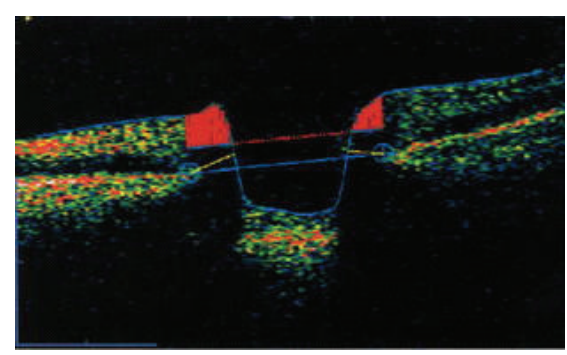

Nerve from Alzheimer's

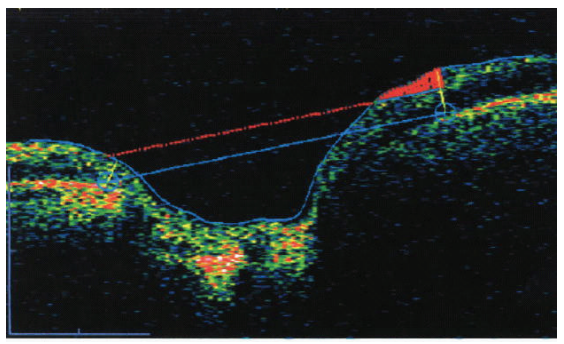

Nerve from Parkinson's with glaucoma

FIgURE 1: The top three images depict the nerve fiber layer (NFL) generated by the older technology; Stratus-OCT. The $y$ axis is NFL thickness in microns and the $x$ axis is the circumference of the optic nerve in degrees; TEMP $=$ temporal, $\mathrm{SUP}=$ superior, NAS $=$ nasal and $\mathrm{INF}=$ inferior. None of the participants had a significant refractive error. The top left figure is from a 73 year old control participant with no known risk factors for glaucoma; Caucasian and age matched to the AD participant. The NFL had an average thickness of 101 microns. The top right image was obtained from a 72 year old participant with no known risk factors for glaucoma; Caucasian, with AD. The NFL had an average thickness of 75 microns. The middle image was from a Caucasian participant, with no known risk factors for glaucoma; that had both Parkinson's disease and glaucoma. The average NFL thickness was 58 microns. The yellow arrows show regions of thinning hypothesized to be due to retrograde neurologic degeneration. The red arrow indicates areas of thinning attributed to glaucoma. The bottom three images are cross sections of the optic nerve. Sections of red indicate the nerve fiber layer. The bottom left image is of the control participant, the bottom middle image is of the AD participant and the bottom right image is of the participant with both Parkinson's disease and glaucoma. 
by glaucoma before the disease is detected by conventional visual field testing of the peripheral vision [53, 54]. As AD destroys visual pathway fibers, the result may simply be an earlier expression of the functional losses in glaucoma because there are fewer neural reserves.

\section{Ocular AD-Related Histopathology $[26,30]$}

$\mathrm{AD}$ damages the lateral geniculate nucleus [19] and glaucoma damages the lateral geniculate nucleus early in the respective disease processes [18]. Damage secondary to AD also occurs in the visual association cortex and other higher cortical areas, the primary visual cortex $[16,55]$, and visual association areas [14]. Additional studies have demonstrated deficits in the retinocalcarine pathway [56-60].

Loss of retinal ganglion cells has been identified by several groups. Such losses will be better characterized by imaging with HDR-OCT. A study by Blanks and colleagues [61] studied 16 individuals with $\mathrm{AD}$ with an age range of 7693. There were 19 normal individuals with an age range from 55-91. They found that within the ganglion cell layer, the cells having the largest diameter may have been preferentially affected in AD. The cells in the magnocellular layer are larger diameter cells.

Sadun and Bassi [60] analyzed 3 eyes from AD individuals ranging in age from 76-89 years. There was degeneration of the retinal ganglion cells. They found axonal degeneration upon examining the retrobulbar optic nerves. The degeneration was reported to be more pronounced the more posterior the nerve. This indicated the retinal ganglion cell loss may be secondary to retrograde axonal degeneration. Hinton and colleagues [57] analyzed the optic nerves of 10 individuals with $\mathrm{AD}$ and 10 age-matched normal individuals ages 76-89 years. This study found eight of the ten nerves obtained from participants with $\mathrm{AD}$ compared to the controls were significantly different in fiber count compared to the normal individuals. The level of $\mathrm{AD}$ severity or duration of illness was not documented. The described findings are in contrast to the findings of Curcio and colleagues [56] who examined four $\mathrm{AD}$ eyes with an age range of $67-86$ years as well as the eyes from four age-matched normal individuals. The individuals with $\mathrm{AD}$ had the disease for at least four years and had severe dementia. Curcio and colleagues did not find difference between the eyes from participants with $\mathrm{AD}$ compared to the age-matched control eyes. Davies and colleagues [62] studied $9 \mathrm{AD}$ and 7 normal eyes and found no histopathologic evidence of differences in the retinal ganglion cells between individuals with $\mathrm{AD}$ and age-matched normal individuals. With imaging using UHR-OCT, we can now measure in vivo, without discomfort, but still obtain detailed imaging of structures that parallel common histologic methods, but eliminates artifacts related to tissue movement, cell displacement, cell shrinkage or nerve fiber dissipation. The UHR-OCT allows for repeat measurements of the same participant over time with image reregistration. The older OCT technology had limits in reregistering location.

\section{Retinal Photographs and AD $[26,30]$}

Using photographs, Hedges and colleagues [59] studied the retinal nerve layer of 26 participants in various stages of $\mathrm{AD}$ and 23 age-matched controls with ages of both groups ranging from 52 to 93 . They found a greater amount of nerve fiber loss in the individuals with $\mathrm{AD}$ compared to age-matched normal individuals. There was a trend towards increasing nerve fiber layer abnormalities with progression and duration of the disease. However, it was not statistically significant.

In the retinal photographs of $22 \mathrm{AD}$ participants and 24 normal age-matched participants, Tsai and colleagues [63] found that five of the $A D$ participants had nerve fiber loss compared to one of the control participants. The photographic method showed that there was a significant correlation in degree of segment optic nerve pallor and the duration of $\mathrm{AD}$. Trends were identified in the segment pallor and the mean Alzheimer's disease Assessment Scale (cognitive, no cognitive and total). Duration and severity of $\mathrm{AD}$ probably impact optic nerve findings. Conventional photographic studies that include only early-onset AD may result in conclusions that the optic nerve is not impacted (and therefore no change in the ganglion cells) because the sample studied has not had the progression of the disease to the point where the ganglion cells have been impacted. However, in the study using OCT looking at both AD and MCI, Pacquet and colleagues found no statistical difference between $\mathrm{AD}$ and $\mathrm{MCI}$ indicating that the OCT imaging may be more sensitive to early disease than any previous histologic or photographic method.

\section{Optic Nerve Fiber Imaging and AD with Stratus-OCT [26]}

Parisi and colleagues [5] used the older version of TimeOCT to assess the optic nerve fiber layer thickness in 17 $\mathrm{AD}$ participants and 14 age-matched control participants and found a significant reduction in nerve fiber thickness in the $\mathrm{AD}$ individuals compared to the age-matched control participants. The age range was from 63 to 77 . The study included participants with mild severity of cognitive impairment. OCT has the ability to measure the thickness of the macula.

Early reports using imaging technologies other than OCT failed to show deficits in the nerve fiber layer associated with AD. Scanning laser polarimetry (GDx Nerve Fiber Analyzer) assesses the nerve fiber layer by measuring changes in the polarization due to the bi-refringent properties of the nerve fiber layer. Once aligned, the image is acquired in 0.7 seconds. Kergoat and colleagues [64] used this technique and found no differences in the optic nerve fiber layer in 30 individualswith early-stage $\mathrm{AD}$, compared to age-matched normal individuals. The same group failed to find nerve fiber layer deficits using yet another imaging technology [65]. The technology, scanning laser tomography (Heidelberg Retinal Tomograph-HRT), uses confocal scanning 
lasers to provide real-time three-dimensional images and measurements of the optic disc and surrounding area. Parameters that can be measured include cup area, disc area, cup volume, rim volume, cup/disc area ratio, and rim $\mathrm{dim} /$ disc area. Once aligned, images are obtained in 1.5 seconds.

Iseri and colleagues used OCT imaging technology to measure macular volume [4] and found that of 28 eyes tested in group of Alzheimer's participants there was significant thinning of the macula compared to 30 eyes tested from a group of control participants. Iseri and colleagues also measured thinning of the retinal nerve fiber layer in those with $\mathrm{AD}$ compared to age-matched control participants and that both the macular thinning and nerve fiber layer thinning were correlated with the severity of cognitive function. Berisha and colleagues [66] have reported the only study to have looked at all three groups; glaucoma, $\mathrm{AD}$, and age-matched control participants. The group of glaucoma participants had an average age of $71, \mathrm{AD}$ participants had an average age of 76 , and the age matched control participants had an average age of 72 . They found significant differences between the groups in the superior quadrant thickness of the nerve fiber layer when measured with OCT and abnormal cup to disc ratios in only the glaucoma group. The glaucoma participants had an average of $106 \mathrm{~mm}$ in the superior optic nerve fiber layer; the $\mathrm{AD}$ participants had an average of $90 \mathrm{~mm}$, and the control participants an average of $115 \mathrm{~mm}$. This study demonstrated that the deficits identified with OCT are unique to $\mathrm{AD}$ and are not undiagnosed ocular pathology such as glaucoma. Superior nerve fiber layer deficits were also identified in those with AD compared to normal age-matched control participants in a study by $\mathrm{Lu}$ and colleagues [6]. This study looked at 22 participants with $\mathrm{AD}$ and 22 age-matched control participants. This study, unlike other studies using OCT, found an increase in the cup to disc ratio of participants with $\mathrm{AD}$ compared to agematched control participants as well as inferior nerve layer deficits compared to the age matched control participants. While not an OCT study, Danesh-Meyers and colleagues used the ocular imaging technology using scanning laser Tomography, HRT [67], and also found an increase in cup to disc ratios in addition to the deficits in nerve fiber layers. If intraocular pressure is the only criteria utilized to rule out glaucoma in studies, this may result in the inclusion of a variety of glaucomas: normal tension glaucomas, glaucomas that have a wide fluctuation in pressure, and glaucomas that had at baseline/young age very low pressures that are now within normal, but high normal ranges. An increased cup to disc ratio, also called cupping, is the physical phenomenon hypothesized to be caused by pressure at the posterior of the eye upon the nerve resulting in cell death and subsequently loss of the fibers themselves. The nerve has fewer nerve fibers along the edge or rim of the optic nerve. As more and more fibers are lost, the nerve starts to be hollowed out and then flattened. Figure 1 shows OCT images of the cup to disc ratio in a normal age-matched control participant, a participant with $\mathrm{AD}$, and a participant with Parkinson's disease as well as glaucoma.
Using the older OCT technology, Paquet and colleagues [2] studied a group of participants with both MCI and $\mathrm{AD}$ and looked at 15 noncognitively impaired age-matched participants, 14 participants with mild AD, 12 participants with moderate $\mathrm{AD}$, and 23 participants with mild cognitive impairment. They found that the nerve fiber layer was thinned in those with mild cognitive impairment, mild $\mathrm{AD}$, and moderate to severe AD. They also did not find any significant difference between those with mild $\mathrm{AD}$ and mild cognitive impairment. Even the older OCT imaging technology with its lower resolution has the potential to identify biomarkers of disease prior to substantial impact on the cognitive functions currently used to identify Alzheimer's disease.

The older OCT technology has also been reported to identify deficits in the nerve fiber layer of those with Parkinson's disease compared to age-matched controls [68-70].

\section{Conclusion}

The visual system provides opportunity to use noninvasive clinical tools to identify and monitor biomarkers of neurodegenerative diseases such as $\mathrm{AD}$. The number of those with $\mathrm{AD}$ in the United States is approaching 5.1 million, and this number is expected to reach 13.2 million by the year 2050 [71]. AD is estimated to cost Americans $\$ 100$ billion a year both in direct costs and indirect costs [72]. OCT imaging has demonstrated structural nerve fiber deficits in the eye in early Alzheimer's disease as well as Mild Cognitive Impairment. Imaging with faster technology with significantly higher resolution such as the Fourier/Spectral/High ResolutionOCT may prove to be an efficient, cost-effective means of identifying and monitoring biomarkers of early AD.

\section{Acknowledgments}

Funding and support for Alzheimer's disease or Parkinson's disease related work and activities were received from Forest Laboratories, Fight for Sight Student Research, AARP Gerontology Scholars, and NIH/NEI Disability/Diversity Supplement Parent project RO1 1AG15361 through the Laboratory of Vision and Cognition at Boston University. Technical support was received from Zeiss Meditec.

\section{References}

[1] D. A. Valenti, "Neuroimaging of retinal nerve fiber layer in AD using optical coherence tomography," Neurology, vol. 69, no. 10, p. 1060, 2007.

[2] C. Paquet, M. Boissonnot, F. Roger, P. Dighiero, R. Gil, and J. Hugon, "Abnormal retinal thickness in patients with mild cognitive impairment and Alzheimer's disease," Neuroscience Letters, vol. 420, no. 2, pp. 97-99, 2007.

[3] F. Berisha, G. T. Feke, C. L. Trempe, J. W. McMeel, and C. L. Schepens, "Retinal abnormalities in early Alzheimer's disease," Investigative Ophthalmology and Visual Science, vol. 48, no. 5, pp. 2285-2289, 2007.

[4] P. K. Iseri, O. Altinaş, T. Tokay, and N. Yüksel, "Relationship between cognitive impairment and retinal morphological 
and visual functional abnormalities in Alzheimer disease," Journal of Neuro-Ophthalmology, vol. 26, no. 1, pp. 18-24, 2006.

[5] V. Parisi, R. Restuccia, F. Fattapposta, C. Mina, M. G. Bucci, and F. Pierelli, "Morphological and functional retinal impairment in Alzheimer's disease patients," Clinical Neurophysiology, vol. 112, no. 10, pp. 1860-1867, 2001.

[6] Y. Lu, Z. Li, X. Zhang et al., "Retinal nerve fiber layer structure abnormalities in early Alzheimer's disease: evidence in optical coherence tomography," Neuroscience Letters, vol. 480, no. 1, pp. 69-72, 2010.

[7] P. Jindahra, T. R. Hedges, C. E. Mendoza-Santiesteban, and G. T. Plant, "Optical coherence tomography of the retina: applications in neurology," Current Opinion in Neurology, vol. 23, no. 1, pp. 16-23, 2010.

[8] A. F. Fercher, C. K. Hitzenberger, W. Drexler, G. Kamp, and H. Sattmann, "In vivo optical coherence tomography," American Journal of Ophthalmology, vol. 116, no. 1, pp. 113-114, 1993.

[9] E. A. Swanson, J. A. Izatt, M. R. Hee et al., "In vivo retinal imaging by optical coherence tomography," Optics Letters, vol. 18, no. 21, pp. 1864-1869, 1993.

[10] D. Cruz, Still evolving, OCT technology has revolutionized diagnostics, Refractive Surgery, 2007.

[11] D. Huang, E. A. Swanson, C. P. Lin et al., "Optical coherence tomography," Science, vol. 254, no. 5035, pp. 1178-1181, 1991.

[12] Zeiss, Stratus OCT Model 3000 User Manual, Zeiss, Dublin, Calif, USA, 2004.

[13] Zeiss, Cirrus HD-OCT, 2010, http://www.cirrusoctdemo.com/ CZM_5947_BS_Cirrus_LY15c\%20final.pdf.

[14] A. C. McKee, R. Au, H. J. Cabral et al., "Visual association pathology in preclinical Alzheimer disease," Journal of Neuropathology and Experimental Neurology, vol. 65, no. 6, pp. 621-630, 2006.

[15] N. Gupta, L. C. Ang, L. N. De Tilly, L. Bidaisee, and Y. H. Yücel, "Human glaucoma and neural degeneration in intracranial optic nerve, lateral geniculate nucleus, and visual cortex," British Journal of Ophthalmology, vol. 90, no. 6, pp. 674-678, 2006.

[16] P. R. Hof and J. H. Morrison, "Quantitative analysis of a vulnerable subset of pyramidal neurons in Alzheimer's disease: II. Primary and secondary visual cortex," Journal of Comparative Neurology, vol. 301, no. 1, pp. 55-64, 1990.

[17] N. Chaturvedi, E. T. Hedley-Whyte, and E. B. Dreyer, "Lateral geniculate nucleus in glaucoma," American Journal of Ophthalmology, vol. 116, no. 2, pp. 182-188, 1993.

[18] Y. H. Yücel, Q. Zhang, N. Gupta, P. L. Kaufman, and R. N. Weinreb, "Loss of neurons in magnocellular and parvocellular layers of the lateral geniculate nucleus in glaucoma," Archives of Ophthalmology, vol. 118, no. 3, pp. 378-384, 2000.

[19] G. Leuba and K. Saini, "Pathology of subcortical visual centres in relation to cortical degeneration in Alzheimer's disease," Neuropathology and Applied Neurobiology, vol. 21, no. 5, pp. 410-422, 1995.

[20] C. J. Duffy, S. J. Tetewsky, and H. O’Brien, "Cortical motion blindness in visuospatial AD," Neurobiology of Aging, vol. 21, no. 6, pp. 867-869, 2000.

[21] G. C. Gilmore, H. E. Wenk, L. A. Naylor, and E. Koss, "Motion perception and Alzheimer's disease," Journals of Gerontology, vol. 49, no. 2, pp. P52-P57, 1994.

[22] R. A. Armstrong, "Visual field defects in Alzheimer's disease patients may reflect differential pathology in the primary visual cortex," Optometry and Vision Science, vol. 73, no. 11, pp. 677-682, 1996.
[23] G. L. Trick, L. R. Trick, P. Morris, and M. Wolf, "Visual field loss in senile dementia of the Alzheimer's type," Neurology, vol. 45, no. 1, pp. 68-74, 1995.

[24] A. Cronin-Golomb, J. F. Rizzo, S. Corkin, and J. H. Growdon, "Visual function in Alzheimer's disease and normal aging," Annals of the New York Academy of Sciences, vol. 640, pp. 2835, 1991.

[25] H. A. Quigley, E. M. Addicks, and W. R. Green, "Optic nerve damage in human glaucoma. III. Quantitative correlation of nerve fiber loss and visual field defect in glaucoma, ischemic neuropathy, papilledema, and toxic neuropathy," Archives of Ophthalmology, vol. 100, no. 1, pp. 135-146, 1982.

[26] D. A. Valenti, "Alzheimer's disease: visual system review," Optometry, vol. 81, no. 1, pp. 12-21, 2010.

[27] A. U. Bayer and F. Ferrari, "Severe progression of glaucomatous optic neuropathy in patients with Alzheimer's disease," Eye, vol. 16, no. 2, pp. 209-212, 2002.

[28] A. J. Weber, H. Chen, W. C. Hubbard, and P. L. Kaufman, "Experimental glaucoma and cell size, density, and number in the primate lateral geniculate nucleus," Investigative Ophthalmology and Visual Science, vol. 41, no. 6, pp. 1370-1379, 2000.

[29] Y. H. Yücel, Q. Zhang, R. N. Weinreb, P. L. Kaufman, and N. Gupta, "Atrophy of relay neurons in magno- and parvocellular layers in the lateral geniculate nucleus in experimental glaucoma," Investigative Ophthalmology and Visual Science, vol. 42, no. 13, pp. 3216-3222, 2001.

[30] D. Valenti, "Anterior visual system and circadian function with reference to Alzheimer's disease," in Vision and Alzheimer's Disease, Interdisciplinary Topics in Gerontology, vol. 34, pp. 129, 2004.

[31] D. L. Bliwise, J. S. Carroll, K. A. Lee, J. C. Nekich, and W. C. Dement, "Sleep and sundowning in nursing home patients with dementia," Psychiatry Research, vol. 48, no. 3, pp. 277292, 1993.

[32] D. G. Harper, E. G. Stopa, A. C. McKee et al., "Differential circadian rhythm disturbances in men with Alzheimer disease and frontotemporal degeneration," Archives of General Psychiatry, vol. 58, no. 4, pp. 353-360, 2001.

[33] J. T. Little, A. Satlin, T. Sunderland, and L. Volicer, "Sundown syndrome in severely demented patients with probable Alzheimer's disease," Journal of Geriatric Psychiatry and Neurology, vol. 8, no. 2, pp. 103-106, 1995.

[34] M. Mirmiran, D. F. Swaab, J. H. Kok, M. A. Hofman, W. Witting, and W. A. Van Gool, "Circadian rhythms and the suprachiasmatic nucleus in perinatal development, aging and Alzheimer's disease," Progress in Brain Research, vol. 93, pp. 151-163, 1992.

[35] D. F. Swaab, E. Fliers, and T. S. Partiman, "The suprachiasmatic nucleus of the human brain in relation to sex, age and senile dementia," Brain Research, vol. 342, no. 1, pp. 37-44, 1985.

[36] A. Buguet, P. Py, and J. P. Romanet, "24-Hour (nyctohemeral) and sleep-related variations of intraocular pressure in healthy white individuals," American Journal of Ophthalmology, vol. 117, no. 3, pp. 342-347, 1994.

[37] A. M. Komaromy, D. E. Brooks, P. S. Kubilis et al., "Diurnal intraocular pressure curves in healthy rhesus macaques (Macaca mulatta) and rhesus macaques with normotensive and hypertensive primary open-angle glaucoma," Journal of Glaucoma, vol. 7, no. 2, pp. 128-131, 1998.

[38] J. H. K. Liu, D. F. Kripke, R. E. Hoffman et al., "Elevation of human intraocular pressure at night under moderate illumination," Investigative Ophthalmology and Visual Science, vol. 40, no. 10, pp. 2439-2442, 1999. 
[39] S. C. Saccà, M. Rolando, A. Marletta, A. Macri, P. Cerqueti, and G. Ciurlo, "Fluctuations of intraocular pressure during the day in open-angle glaucoma, normal-tension glaucoma and normal subjects," Ophthalmologica, vol. 212, no. 2, pp. 115119,1998

[40] C. Noël, A. M. Kabo, J. P. Romanet, A. Montmayeur, and A. Buguet, "Twenty-four-hour time course of intraocular pressure in healthy and glaucomatous Africans: relation to sleep patterns," Ophthalmology, vol. 108, no. 1, pp. 139-144, 2001.

[41] M. Münch, S. Kobialka, R. Steiner, P. Oelhafen, A. WirzJustice, and C. Cajochen, "Wavelength-dependent effects of evening light exposure on sleep architecture and sleep EEG power density in men," American Journal of Physiology, vol. 290, no. 5, pp. R1421-R1428, 2006.

[42] D. M. Berson, F. A. Dunn, and M. Takao, "Phototransduction by retinal ganglion cells that set the circadian clock," Science, vol. 295, no. 5557, pp. 1070-1073, 2002.

[43] A. U. Bayer, F. Ferrari, and C. Erb, "High occurrence rate of glaucoma among patients with Alzheimer's disease," European Neurology, vol. 47, no. 3, pp. 165-168, 2002.

[44] A. U. Bayer and C. Erb, "Short wavelength automated perimetry, frequency doubling technology perimetry, and pattern electroretinography for prediction of progressive glaucomatous standard visual field defects," Ophthalmology, vol. 109, no. 5, pp. 1009-1017, 2002.

[45] H. Tamura, H. Kawakami, T. Kanamoto et al., "High frequency of open-angle glaucoma in Japanese patients with Alzheimer's disease," Journal of the Neurological Sciences, vol. 246, no. 1-2, pp. 79-83, 2006.

[46] J. P. Berdahl and R. R. Allingham, "Intracranial pressure and glaucoma," Current Opinion in Ophthalmology, vol. 21, no. 2, pp. 106-111, 2010.

[47] W. H. Morgan, C. Balaratnasingam, S. J. Cringle, and D. Y. Yu, "Glaucoma and cerebrospinal fluid pressure," Ophthalmology, vol. 115, no. 12, pp. 2317-2318, 2008.

[48] H. E. Killer, J. Flammer, and N. R. Miller, "Glaucoma and cerebrospinal fluid pressure," Ophthalmology, vol. 115, no. 12, pp. 2316-2317, 2008.

[49] J. P. Berdahl, R. R. Allingham, and D. H. Johnson, "Cerebrospinal fluid pressure is decreased in primary open-angle glaucoma," Ophthalmology, vol. 115, no. 5, pp. 763-768, 2008.

[50] J. B. Jonas, E. Berenshtein, and L. Holbach, "Anatomic relationship between lamina cribrosa, intraocular space, and cerebrospinal fluid space," Investigative Ophthalmology and Visual Science, vol. 44, no. 12, pp. 5189-5195, 2003.

[51] P. Wostyn, K. Audenaert, and P. P. De Deyn, "Alzheimer's disease-related changes in diseases characterized by elevation of intracranial or intraocular pressure," Clinical Neurology and Neurosurgery, vol. 110, no. 2, pp. 101-109, 2008.

[52] P. Wostyn, K. Audenaert, and P. P. De Deyn, "An abnormal high trans-lamina cribrosa pressure difference: a missing link between Alzheimer's disease and glaucoma?" Clinical Neurology and Neurosurgery, vol. 110, no. 7, pp. 753-754, 2008.

[53] H. A. Quigley, E. M. Addicks, and W. R. Green, "Optic nerve damage in human glaucoma. III. Quantitative correlation of nerve fiber loss and visual field defect in glaucoma, ischemic neuropathy, papilledema, and toxic neuropathy," Archives of Ophthalmology, vol. 100, no. 1, pp. 135-146, 1982.

[54] R. S. Harwerth, L. Carter-Dawson, F. Shen, E. L. Smith, and M. L. J. Crawford, "Ganglion cell losses underlying visual field defects from experimental glaucoma," Investigative Ophthalmology and Visual Science, vol. 40, no. 10, pp. 22422250, 1999.

[55] P. Pietrini, M. L. Furey, N. Graff-Radford et al., "Preferential metabolic involvement of visual cortical areas in a subtype of Alzheimer's disease: clinical implications," American Journal of Psychiatry, vol. 153, no. 10, pp. 1261-1268, 1996.

[56] C. A. Curcio and D. N. Drucker, "Retinal ganglion cells in Alzheimer's disease and aging," Annals of Neurology, vol. 33, no. 3, pp. 248-257, 1993.

[57] D. R. Hinton, A. A. Sadun, J. C. Blanks, and C. A. Miller, "Optic-nerve degeneration in Alzheimer's disease," The New England Journal of Medicine, vol. 315, no. 8, pp. 485-487, 1986.

[58] J. C. Blanks, D. R. Hinton, A. A. Sadun, and C. A. Miller, "Retinal ganglion cell degeneration in Alzheimer's disease," Brain Research, vol. 501, no. 2, pp. 364-372, 1989.

[59] T. R. Hedges, R. P. Galves, D. Speigelman, N. R. Barbas, E. Peli, and C. J. Yardley, "Retinal nerve fiber layer abnormalities in Alzheimer's disease," Acta Ophthalmologica Scandinavica, vol. 74, no. 3, pp. 271-275, 1996.

[60] A. A. Sadun and C. J. Bassi, "Optic nerve damage in Alzheimer's disease,” Ophthalmology, vol. 97, no. 1, pp. 9-17, 1990.

[61] J. C. Blanks, S. Y. Schmidt, Y. Torigoe, K. V. Porrello, D. R. Hinton, and R. H. I. Blanks, "Retinal pathology in Alzheimer's disease. II. Regional neuron loss and glial changes in GCL," Neurobiology of Aging, vol. 17, no. 3, pp. 385-395, 1996.

[62] D. C. Davies, P. McCoubrie, B. McDonald, and K. A. Jobst, "Myelinated axon number in the optic nerve is unaffected by Alzheimer's disease," British Journal of Ophthalmology, vol. 79, no. 6, pp. 596-600, 1995.

[63] C. S. Tsai, R. Ritch, B. Schwartz et al., "Optic nerve head and nerve fiber layer in Alzheimer's disease," Archives of Ophthalmology, vol. 109, no. 2, pp. 199-204, 1991.

[64] H. Kergoat, M. J. Kergoat, L. Justino, H. Chertkow, A. Robillard, and H. Bergman, "An evaluation of the retinal nerve fiber layer thickness by scanning laser polarimetry in individuals with dementia of the Alzheimer type," Acta Ophthalmologica Scandinavica, vol. 79, no. 2, pp. 187-191, 2001.

[65] H. Kergoat, M. J. Kergoat, L. Justino, A. Robillard, H. Bergman, and H. Chertkow, "Normal optic nerve head topography in the early stages of dementia of the Alzheimer type," Dementia and Geriatric Cognitive Disorders, vol. 12, no. 6, pp. 359-363, 2001.

[66] F. Berisha et al., "Localized retinal nerve fiber layer thinning in patients with early Glaucoma or Alzheimer's disease," Investigative Ophthalmology \& Visual Science, vol. 47, 2006, Abstract no. 3379.

[67] H. V. Danesh-Meyer, H. Birch, J. Y. F. Ku, S. Carroll, and G. Gamble, "Reduction of optic nerve fibers in patients with Alzheimer disease identified by laser imaging," Neurology, vol. 67, no. 10, pp. 1852-1854, 2006.

[68] O. Altintaş, P. Işeri, B. Ozkan, and Y. Cağlar, "Correlation between retinal morphological and functional findings and clinical severity in Parkinson's disease," Documenta Ophthalmologica, vol. 116, no. 2, pp. 137-146, 2008.

[69] R. Inzelberg, J. A. Ramirez, P. Nisipeanu, and A. Ophir, "Retinal nerve fiber layer thinning in Parkinson disease," Vision Research, vol. 44, no. 24, pp. 2793-2797, 2004.

[70] G. F. Yavas, O. Yilmaz, T. Küsbeci, and F. Oztürk, "The effect of levodopa and dopamine agonists on optic nerve head in Parkinson disease," European Journal of Ophthalmology, vol. 17, no. 5, pp. 812-816, 2007. 
[71] L. E. Hebert, P. A. Scherr, J. L. Bienias, D. A. Bennett, and D. A. Evans, "Alzheimer disease in the US population: prevalence estimates using the 2000 census," Archives of Neurology, vol. 60, no. 8, pp. 1119-1122, 2003.

[72] R. L. Ernst and J. W. Hay, "The US economic and social costs of Alzheimer's disease revisited," American Journal of Public Health, vol. 84, no. 8, pp. 1261-1264, 1994. 


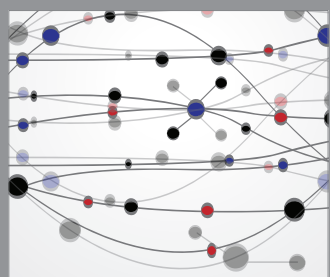

The Scientific World Journal
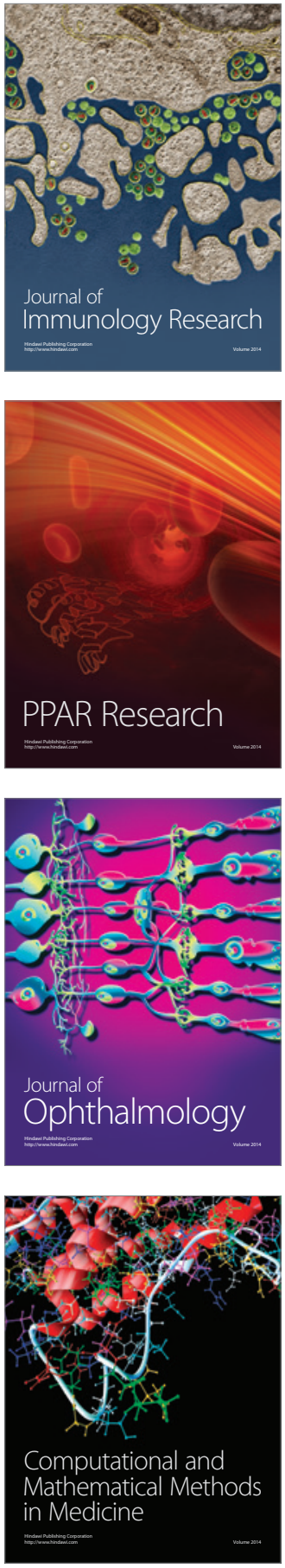

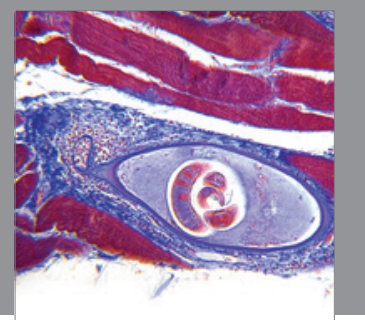

Gastroenterology

Research and Practice
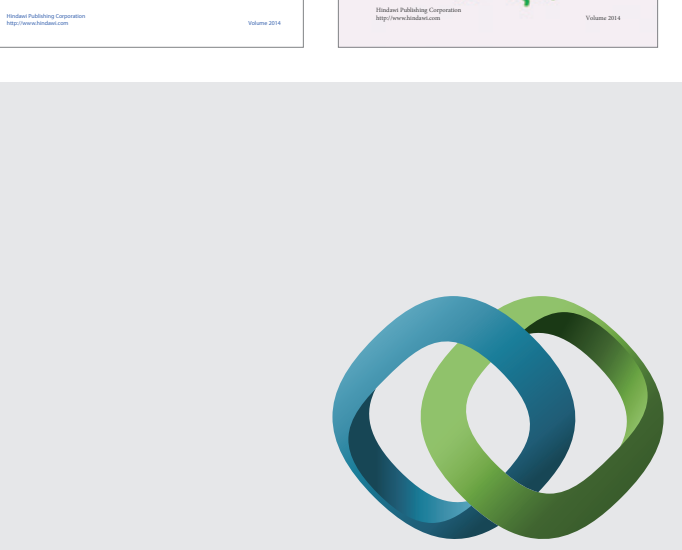

\section{Hindawi}

Submit your manuscripts at

http://www.hindawi.com
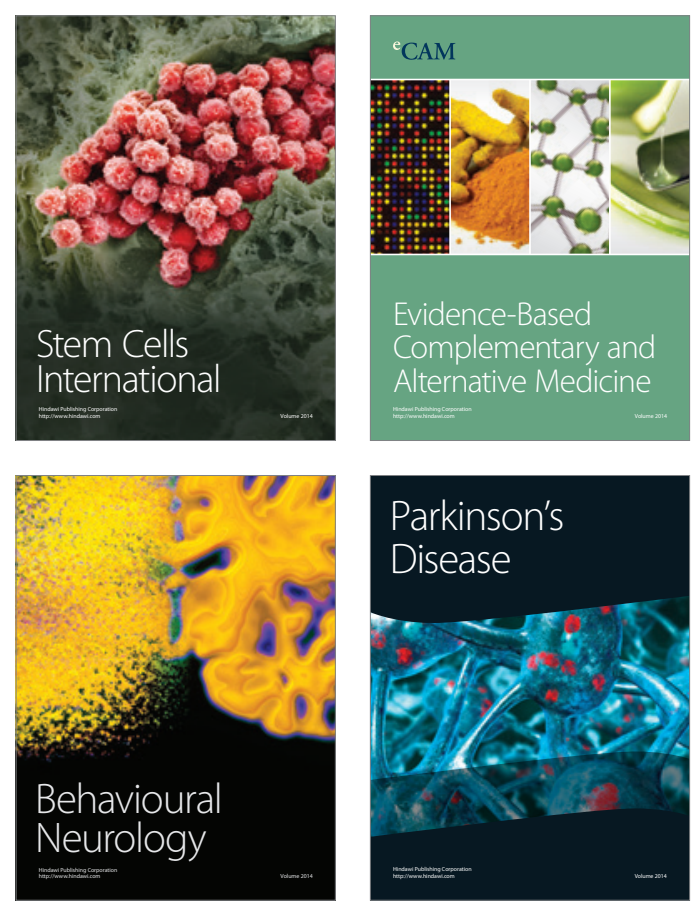

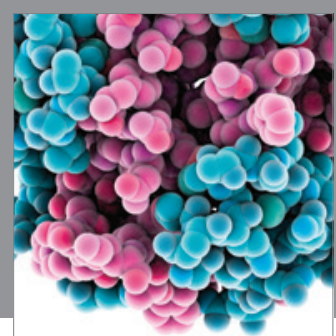

Journal of
Diabetes Research

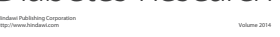

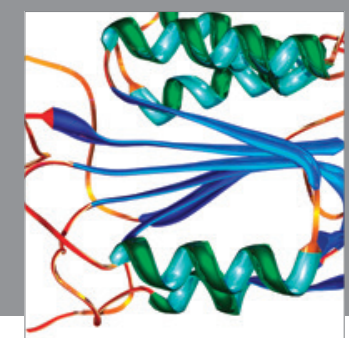

Disease Markers
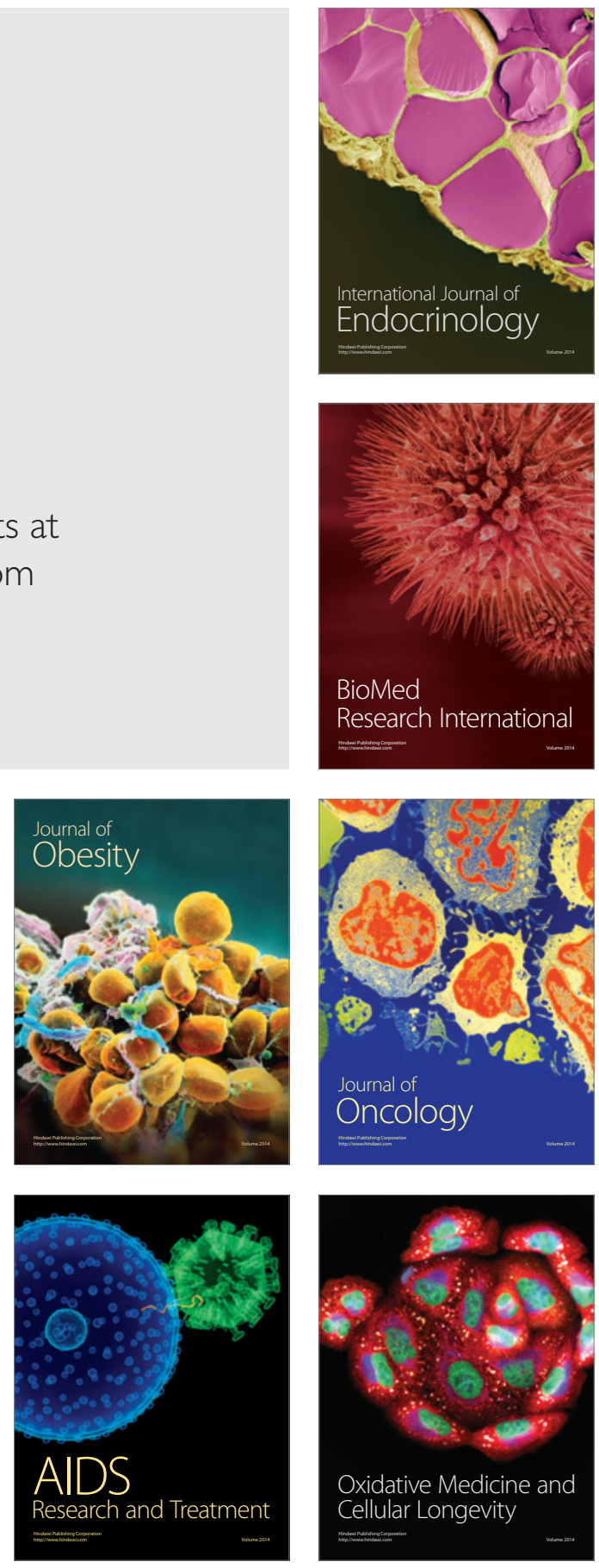